This is a postprint version of the following published document: Noemí Merayo; Juan Carlos Aguado; Carlos Marcos Miguel; Ramón José Durán; Ignacio de Miguel; Patricia Fernández; Rubén Mateo Lorenzo; Evaristo José Abril. Testbed laboratory for the physical analysis of gigabit passive optical access networks (GPONs) .In: 2017 56th FITCE Congress. DOI: https://doi.org/ 10.1109/FITCE.2017.8093009

(C) 2017 IEEE. Personal use of this material is permitted. Permission from IEEE must be obtained for all other uses, in any current or future media, including reprinting/republishing this material for advertising or promotional purposes, creating new collective works, for resale or redistribution to servers or lists, or reuse of any copyrighted component of this work in other works. 


\title{
Testbed Laboratory for the Physical Analysis of Gigabit Passive Optical Access Networks (GPONs)
}

\author{
Noemí Merayo, Juan Carlos Aguado, Carlos Marcos Miguel, Ramón José Durán, Ignacio de Miguel, Patricia \\ Fernández, Rubén Mateo Lorenzo, Evaristo José Abril \\ Optical Communications Group, Department of Signal Theory, Communications and Telematic Engineering. \\ E.T.S.I. Telecomunicación, Universidad de Valladolid (Spain), Campus Miguel Delibes, Paseo de Belén 15, 47011 \\ Valladolid, Spain, noemer@tel.uva.es
}

\begin{abstract}
Telecom engineering students should face important challenges in their careers regarding next-generation networks. In fact, in the last few years optical access networks are leading an important revolution in the network sector. Passive Optical Networks (PONs) based on the Ethernet (EPON) and the Gigabit (GPON) protocols are the most deployed optical access networks around the world. Therefore, Universities should join to this revolution permitting their students to train and to learn these innovative and recent technologies so that they have the chance to develop and to improve some required skills for their future. Consequently, we deployed a real GPON testbed in a laboratory where students can research. In the first approach students have to analyze issues regarding the physical layer of the GPON testbed. In fact, they have to test eye diagrams, $Q$ factors, rise/fall times of transmitters and extinction ratios. Furthermore, they have to discover and analyze the events (connectors, fusion splices, attenuation) at the optical links of the access network using an Optical Time-Domain Reflectometer (OTDR). Besides, the GPON is being prepared so that students can deal in other lab sessions with its configuration and management.
\end{abstract}

Keywords-Textbed; Physical analysis; Gigabit Passive Optical Networks; testbed; eye diagram, Optical Time-Domain Reflectometer (OTDR); Digital oscilloscope

\section{INTRODUCTION}

Future telecom engineers need to acquire special learning and training skills regarding next-generation access and core networks based on fiber technologies. Recently, the access network segment based on optical fiber is gaining great importance due to their massive deployment around the world. This phenomenon has happened because traditional access technologies, based on coaxial and copper became so obsolete that they led a big bottleneck that affected the QoS (Quality of Service) requirements in the end-to-end network. In this way, Passive Optical Networks (PONs) are the most important deployed access network architectures with an estimation of 22 million homes connected by the end of 2018 in Europe. Besides, 100 million people in the Asia-Pacific region are now subscribed to Internet services by means of PON infrastructures [1]. In a typical configuration a Passive Optical Network follows a tree topology between the Optical Line Terminal (OLT), located in the Central Office, and an Optical Network Terminal (ONT), located at the user's house (Fig. 1).

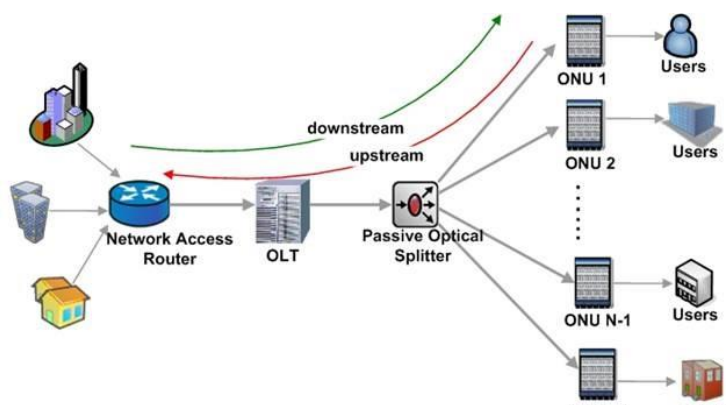

Figure 1. Tree topology of a typical PON architecture

The connection between the OLT and the ONUs is done by means of an optical splitter [2-3]. These access networks use optical fiber as the transmission medium and they are provided with two channels (two independent wavelengths). In the downstream channel $(1490 \mathrm{~nm})$, the connectivity is point-to-multipoint between the OLT and the ONTs. In contrast, in the upstream channel $(1310 \mathrm{~nm})$, from ONTs to the OLT, PONs have a multipoint-to-point connectivity so every ONU share the same wavelength. As a consequence, a MAC (Medium Access Control) protocol is required to manage data collisions of different users (ONTs) [4].

Therefore, these access networks can be analyzed from two different points of views. On the one hand, the QoS and the efficient management of the network resources have become one key point in PONs, especially with the new emerging services and applications. In particular, the end-toend delay and the allocated bandwidth are becoming important limiting factors for the user experience. On the other hand, the study of the physical layer at both channels (upstream and downstream) and the impact of physical impairments in the access architecture become crucial for the good performance of a PON. As a consequence, we have deployed a real tested of a GPON (based on the Gigabit standard) in one laboratory of the School of Telecommunications so that students can train with a real access network scenario. Once we build the GPON network of $25 \mathrm{~km}$ with every connected device (OLT, passive splitter, ONTs), we configured the GPON emulating a network provider, by means of providing different QoS services and priority user profiles. In fact, in this paper we describe some lab sessions regarding the physical analysis of GPON architectures and how students learn and train with physical concepts in a real optical access network. 


\section{DESCRIPTION OF THE REAL GPON TESTBED IN THE OPTICAL COMMUNICATION LABORATORY}

With the aim of acquiring some important skills and optical communication knowledge, a real testbed composed of a Gigabit Passive Optical Access Network (GPON) has been deployed in the Optical Communication laboratory of the School of Telecommunications, as it can be observed in Fig. 2.

The GPON is implemented using equipment from the Telnet-RI vendor [5]. In particular, we use an OLT SmartOLT 350 (located at the central office), which implements a full-duplex GPON interface of $2.488 \mathrm{Gbps}$ (downstream) and 1.244 Gbps (upstream), being fully compliant with ITU-T G.984.1-4 [6] and G.988 specifications [7]. It includes 4 ports, each supporting up to 64 ONTs. Moreover, it also complies with 802.1q and 802.ad standards, thus enabling $\mathrm{C}$ and $\mathrm{S}$ tagging to provide QoS requirements. Furthermore, the connection between the OLT and the optical splitter includes three spools of Standard Single Mode Fiber (SSMF) of different lengths (two of $5 \mathrm{~km}$ and one of $10 \mathrm{~km}$ ), thereby permitting to configure the reach of the network to $5,10,15$ or $20 \mathrm{~km}$ (plus the length of the distribution fiber, as later described). The testbed is also equipped with two optical splitters 1:8, thus enabling the configuration of a two-stage splitting topology if desired.

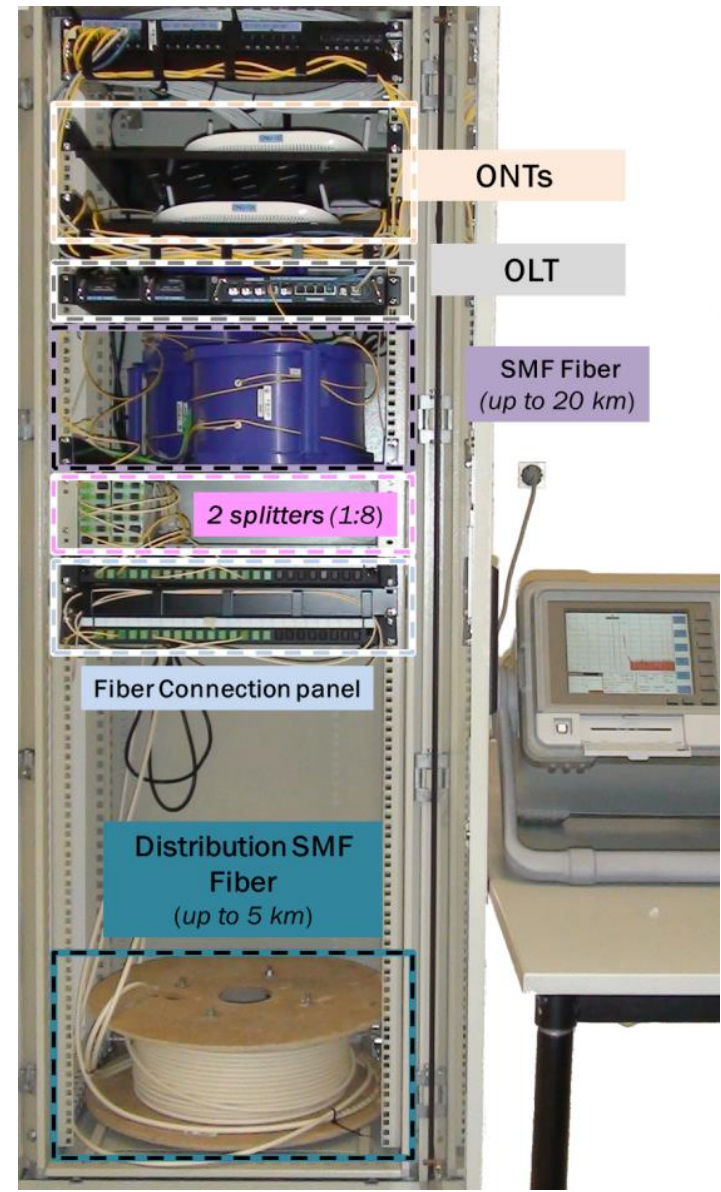

Figure 2. Real deployment of the GPON testbed in the Optical Communications laboratory
Then, the splitters (passive couplers) are connected to the ONTs by means of distribution fibers. The length of each link can be individually configured, by means of a connection panel, from 100 meters up to $5 \mathrm{~km}$. In this way, the testbed can be used to analyze realistic scenarios where different users have different delays (different distances) from their location to the central office. Finally, at this moment, the testbed employs L3 (level 3) model ONTs (Wave Access 3021) [5], which means that they integrate router functionalities. Those ONTs comply with the ITU-T G.984.x [6-7] specifications, supporting 2.488 Gbps (downstream) and 1.244 Gbps (upstream).

\section{DESCRIPTION OF THE METHODOLOGY OF THE LABORATORY SESSIONS}

To assess the benefits of the GPON testbed, we use it in a set of subjects of different courses and degrees. In particular, students face the physical analysis of optical fibers and systems in subjects that belong to the third course of two four years degrees. The subjects, Guided Communication Systems (GCS) and Optical Communications (OC) [8], are taught in the second semester and they are compulsory, with a total of 32 students. All subjects share the same objectives and the basic knowledge and skills that students should acquire at the end of the subjects are the next [9-10]:

- To know the principles of the fiber propagation.

- To know the main characteristics of basic optical components and devices.

- To describe the most important optical fiber propagation problems.

- To design optical network systems in the physical layer.

- To efficiently manage optical instrumentation and optical network simulators to carry out experiments to analyze fiber parameters and network performance at the physical layer.

Then, both subjects consist of lectures and laboratory sessions regarding theoretical and experimental optical concepts. The laboratory consists of two different parts. In one part, students have to deal with the network simulation platform Optsim, a software tool developed by RSoft [11] to design and simulate optical communication systems. This simulation platform is highly spread in the optics research field, but also it is used for teaching purposes in qualified subjects. During these lab sessions students analyze the performance of different optical modulation techniques and fibers, as well as physical phenomena such as the attenuation and the dispersion in optical systems. In the second part, students make fiber connectors to provide pluggable connections between optical devices and also fusion splicing of optical fibers using an automatic optical splicer (to fuse fibers using heat). Furthermore, students learn to use an OTDR, an optoelectronic instrument to characterize optical networks. Indeed, student will use this optical device to 
analyze the GPON testbed along 25 kilometers at both optical links, the upstream and the downstream channel.

On the other hand, the GPON network is being preparing to be used in two more specialized subjects of the fourth course. These subjects, Optical Communications Systems and Networks (OCSN) and Optical Communications Systems (OCS), are both taught in the second semester with a total of 25 students. In these advanced subjects students have to deal with the QoS and the efficient management of network resources in the GPON.

\section{SUMMARY OF EXPERIMENTS PERFORMED BY STUDENTS IN THE PHYSICAL LAYER}

In this section we describe the experiments performed by students to train with the GPON testbed analyzing different issues of the physical layer using a set of optical devices. In Fig. 3, we show the GPON testbed with the optical instrumentation that students have to use during the lab sessions in the optical communications laboratory.

\section{A. OTDR to analyse the physical upstream and downstream GPON channels}

The OTDR is an optoelectronic instrument used to characterize optical fiber networks. It injects a series of optical pulses into the fiber under test and extracts, from the same end of the fiber, light that is reflected back (scattered) from points along the fiber (Rayleigh backscatter phenomena). The reflected light is used to characterize the optical fiber [12]. The strength of the return pulses is measured and plotted as a function of the fiber length, as it can be observed in Fig. 4. Then, the OTDR helps us to check if the optical links of one network comply with the minimum quality requirements. These requirements can be related to the accumulative attenuation in the optical link or to detect if there is any problem at any place of the link.

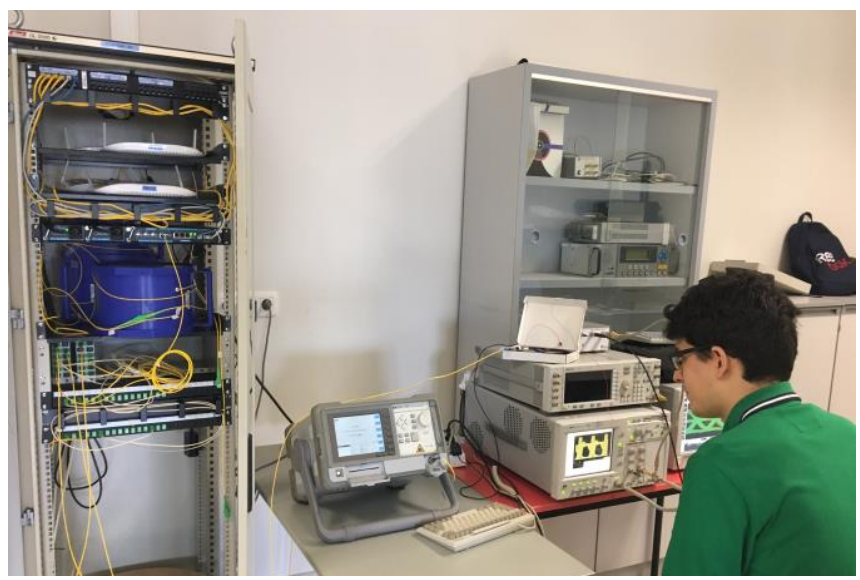

Figure 3. The GPON testbed and the optical instrumentation used in the optical communication laboratory

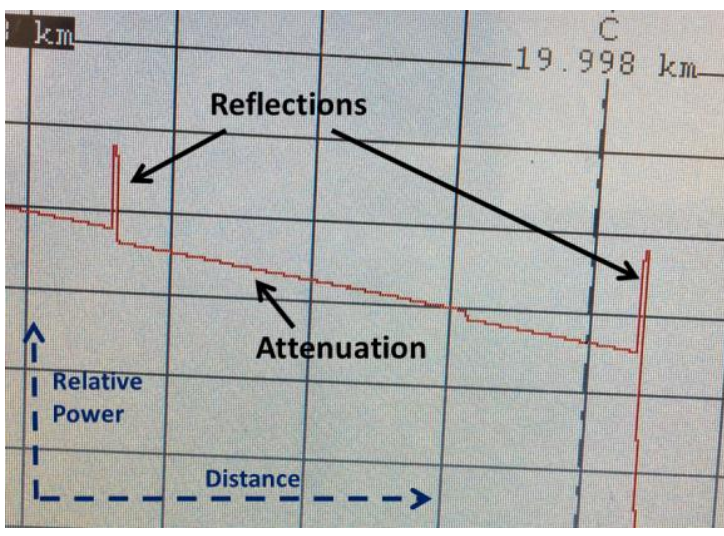

Figure 4. Events captured by the OTDR in an optical link

Therefore, this optoelectronic device visually represents information of every detected event in the optical link, such as the location, length and state of splices, connectors or fusion splices. Even more, the OTDR is able to detect cuts or abnormal performance of the optical fiber and their location in the link. In the Optical Communications laboratory we have the Hewlett-Packard 8147A Optical Time Domain Reflectometer [12] for single mode optical fibers. As a consequence, the main objectives to achieve in these lab sessions using the OTDR instrument in the GPON testbed can be summarized as:

- To familiarize with the OTDR device.

- To configure the OTDR to efficiently obtain information of one fiber installation with unknown events (GPON testbed).

- To locate and to interpreter the different events visualized by the OTDR along the $25 \mathrm{~km}$ of the GPON at the upstream channel $(1310 \mathrm{~nm})$. Students should analyze the performance using different pulses lengths (in nanometers and micrometers).

- To locate and to interpreter events along the $25 \mathrm{~km}$ of the GPON at the downstream channel $(1490 \mathrm{~nm})$, as it be observed in Fig. 5 .

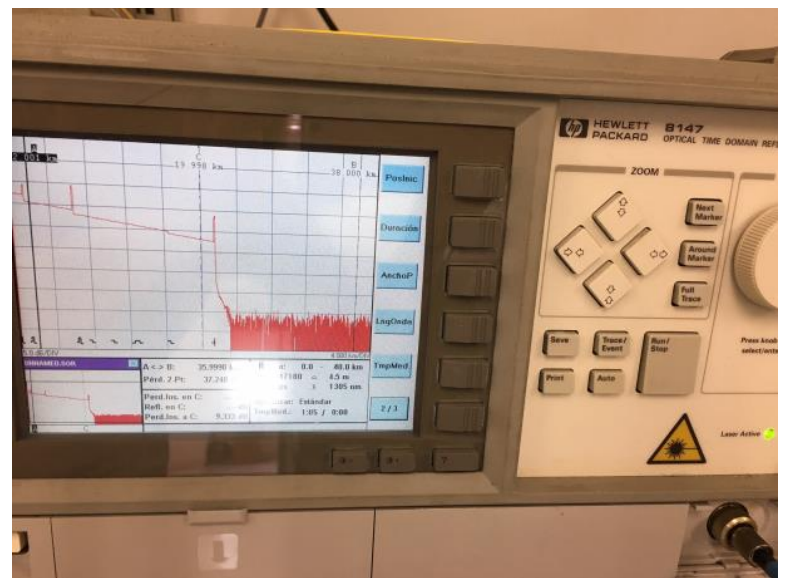

Figure 5. Visualization of different events in the donwstream channel of the GPON along the $25 \mathrm{~km}$ 


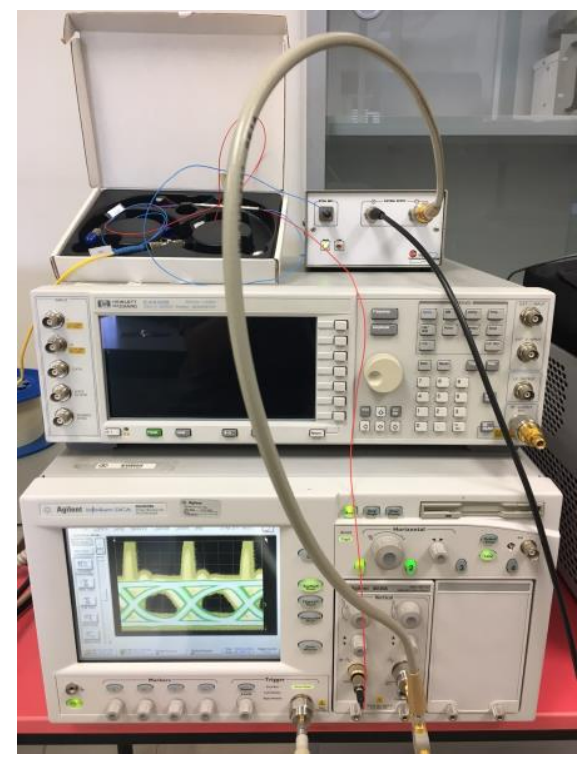

Figure 6. Connection of the ouptut signal of the OLT to the digital oscilloscope to visualize the optical and electrical eye pattners.

\section{B. Eye Patterns and Mask Test on Digital Oscilloscopes}

In this part of the lab sessions students have to deal with some optical quality metrics. In particular, students have to test the optical and the electrical signals of the OLT (located at the Central Office) and the signal transmitted by the ONTs (located at the user's side) using some metrics such as the mask test, the eye diagram, the $\mathrm{Q}$ factor, the extinction ratio or the rise/fall times of transmitters. In the next sub-sections we describe the experimental investigation regarding these issues. In order to carry out these experiments, students will use the digital storage oscilloscopes of Agilent model DSO90254A [13] and model 86100B [14].

1) Optical and electrical conversion signal at the output of the OLT: To carried out this analysis we connect the OLT to the oscillocope at a data rate of $2.488 \mathrm{Gpbs}$. In this laboratoty setup students compare both, the optical and the electrical eye diagram, so we have to conect the output of the OLT to a 50:50 optical coupler (Fig. 6). One optical output of the coupler directly goes to the oscilloscope (Channel 1) and the other output goes to the electrical receiver to be converted to electrical. Finally, the output signal of the receiver (electrical) is connected to Channel 2 of the oscillocope.

The experimental research based on the complete equipment setup permits students:

- To compare the optical and the electrical eye diagram, analyzing for example the overshooting effect of the laser of the OLT, as it can be observed in Fig. 7.

- To measure and to compare rise/fall times, pulse width, extinction ratio or jitter from the eye patterns at the output of the OLT (Fig. 7).

- To measure the Q factor and the BER (Bit Error Rate) at the output of the OLT.

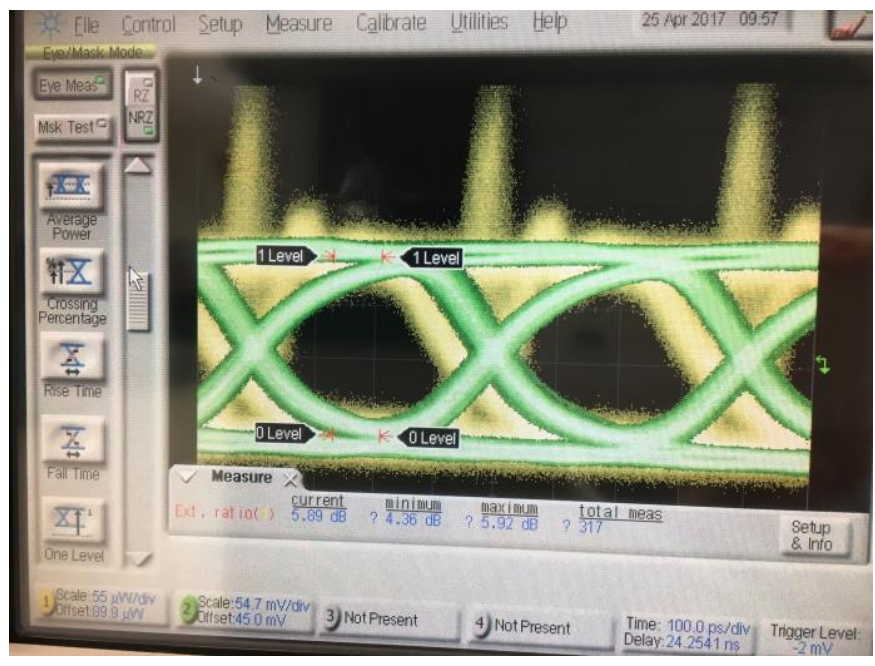

Figure 7. Visualization and comparison of both the optical and the electrical eye diagram at the output of the OLT.

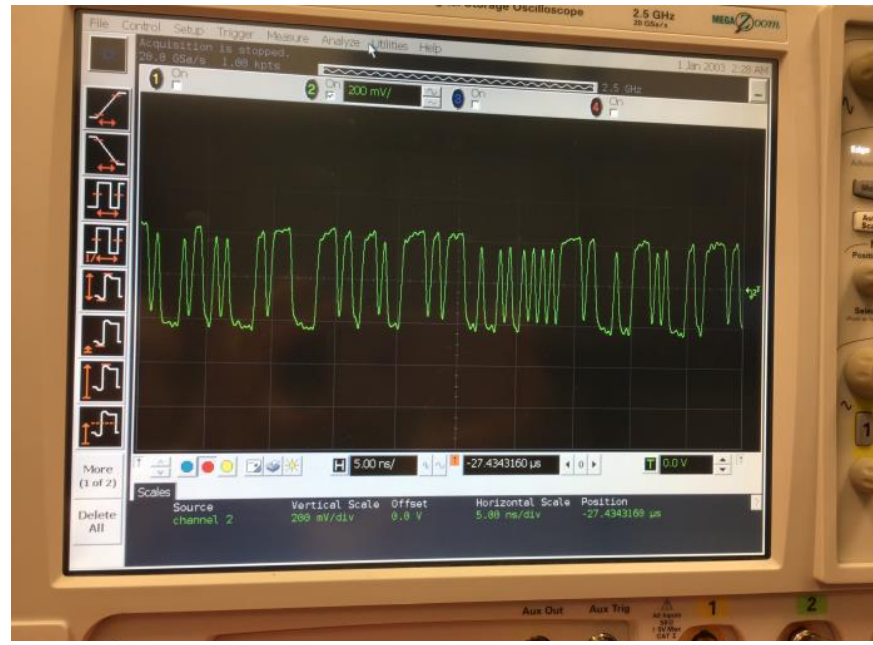

Figure 8. Visualization of the data frame with the information transmitted by the OLT.

- To compare the theoretical eye mask of the GPON standard with the one obtained in the oscilloscope.

- To visualize the data frame transmitted by the OLT, as it shown in Fig. 8.

2) To visualize the data frame and the optical power performance of the signal transmitted by the ONTs: In this experiment, students will visualize the data frames transmitted by the connected ONTs. As it can be observed in Fig.9, we can differ data frames from different ONTs, as each of them transmits at a different intantaneous optical power. Moreover, using the oscilloscope students can deeply analyze the data frame, for example they can distinguish the guard time or the preamble of the frames transmitted by the different ONTs, as it shown in Fig. 10. Finally, the digital oscilloscope is able to visualize the eye diagram of every connected ONT. 


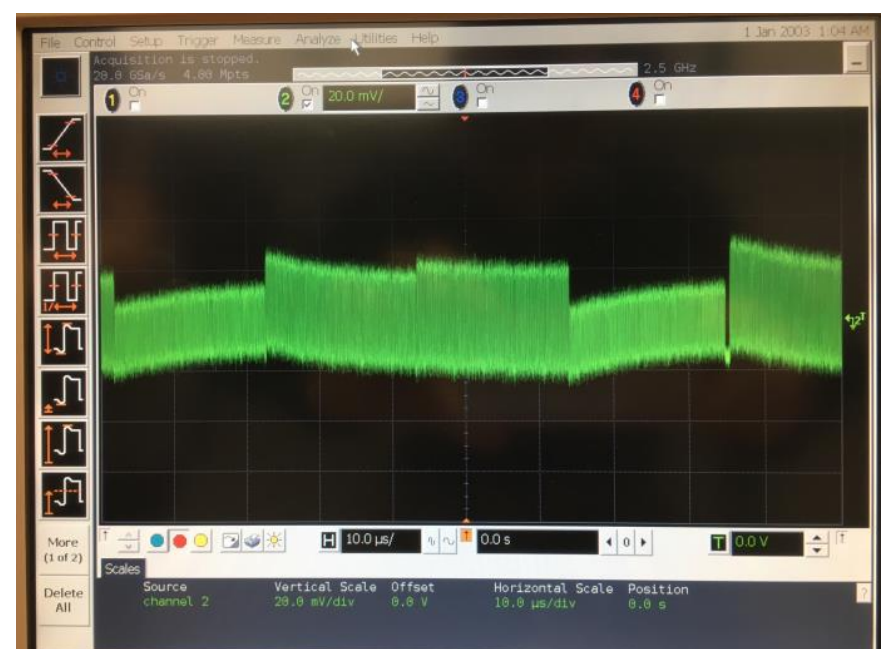

Figure 9. Visualization of the data frames with information transmitted by the connected ONTs.

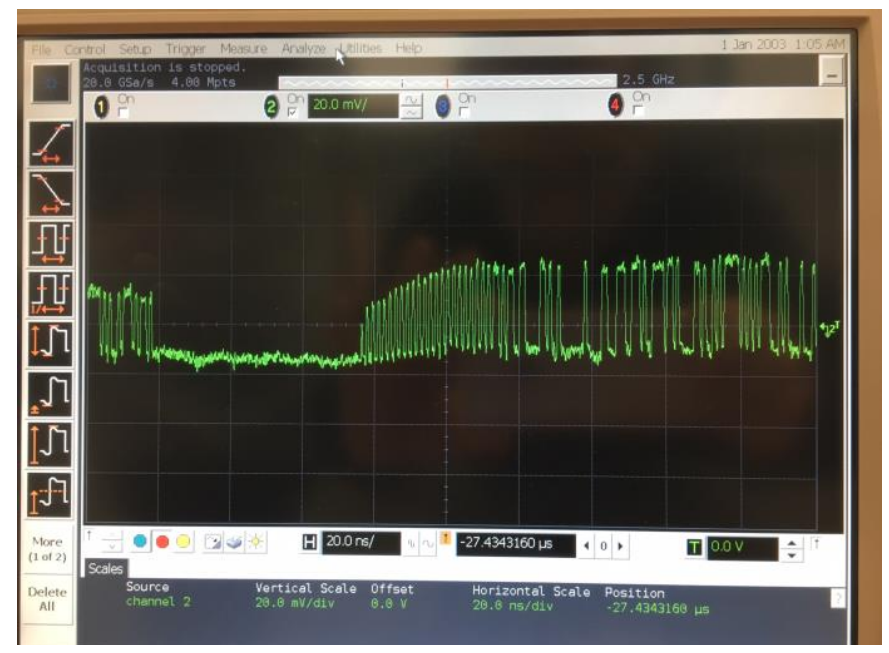

Figure 10. Preamble and guard times of one data frame transmitted by one ONT.

\section{CONCLUSIONS}

In this paper, we have described the deployment and configuration of a real GPON access network in an Optical Communication laboratory. The GPON testbed is used by students in a set of subjects to test its performance in the physical layer and in a near future in the medium access control layer (management and QoS configuration). In fact, the first contact of students with the GPON testbed regards the physical analysis in both channels of the optical network (upstream and downstream direction). In particular, they learn to use the Optical Time Domain Reflectometer to discover the physical events (connectors, fusion splices, splitters, attenuation) along the $25 \mathrm{~km}$ of the end-to-end network. Even more, students have to check the quality of these events and detect any problem in the optical link. Moreover, students learn to configure digital oscilloscopes to analyze the optical and the electrical eye diagrams of the OLT and the ONTs. In this way, they test the Q factor (with their corresponding BER), the rise/fall times of the lasers, the extinction ratio and the data frames transmitted at both sides of the GPON testbed. However, it is worth saying that a set of lab sessions are being preparing so that students can deal with the QoS management and resources allocation in the GPON testbed. These laboratory experiments will provide future telecom engineers with important skills and knowledge regarding the most deployed next-generation optical networks over the world.

\section{ACKNOWLEDGMENT}

This work has been funded by Spanish Ministry of Science and Innovation (TEC2014-53071-C3-2-P and TEC2015-71932-REDT).

\section{REFERENCES}

[1] K. Ahl, "FTTH Council Europe. Creating Connected Continents. Bringing people together through FTTH," http://www.ftthcouncil.eu/documents/Publications/TLA6.pdf, Accessed April 2017.

[2] M. Pesavento and A. Kelsey, "PONs for the Broadband Local Loop," Lightwave, vol. 16, Sept. 1999, pp. 68-74.

[3] B. Lung, "Fiber to the Home Using a PON Infrastructure," IEEE/OSA Journal of Lightwave Technology ,vol. 2, Dec. 2006, pp. 4568-4583, doi: 10.1109/JLT.2006.885779.

[4] A. Walid, and A. Chen, "Efficient and dynamic bandwidth allocation for Non-Status Reporting Gigabit Passive Optical Networks (GPON)," In Proc. of the 2015 IEEE International Conference on Communications (ICC), 2015, Kuala Lumpur, Malasia, pp. 10001005

[5] Telnet-RI vendor web page, http://www.telnet-ri.es/en, Accessed April 2017.

[6] International Telecommunication Union, "G.984.1 : Gigabit-capable passive optical networks (GPON): General characteristics," http://www.itu.int/rec/T-REC-G.984.1-200803-I, Accessed January 2017.

[7] International Telecommunication Union, "G.988 : ONU management and control interface (OMCI) specification," https://www.itu.int/rec/T-REC-G.988-201210-I, Accessed January 2017.

[8] School of Telecommunications web page, "School of Telecommunications home page", http://www.te.uva.es. Accessed January 2017.

[9] H. Kolimbiris, Fiber Optic Communications, Peason Prentice Hall Education. 2004.

[10] G. P. Agrawal, Fiber Optic Communication Systems, John Wiley and Sons, Inc. New York, 2002.

[11] RSoft Photonic Design Software: Photonic Device \& Optical Communications System Design, https://optics.synopsys.com/rsoft/", Accessed December 2016.

[12] Hewlett-Packard 8147A Optical Time Domain Reflectometer, http://www.rlscientific.com/documenti/E4310A.pdf.

[13] Digital Storage Oscilloscope Agilent Model DSO90254A "https://www.naic.edu/ phil/hardware/oscilloscopes/9000A_Series_ User_Guide_10_2009.pdf," Accessed 10 April 2017.

[14] Digital Storage Oscilloscope Agilent Model 86100B, "http://literature.cdn.keysight.com/litweb/pdf/5989-0278EN.pdf," Accessed 10 April 2017. 